Cosmography with the Einstein Telescope

This article has been downloaded from IOPscience. Please scroll down to see the full text article.

2010 Class. Quantum Grav. 27215006

(http://iopscience.iop.org/0264-9381/27/21/215006)

View the table of contents for this issue, or go to the journal homepage for more

Download details:

IP Address: 194.94.224.254

The article was downloaded on 27/09/2011 at 10:39

Please note that terms and conditions apply. 


\title{
Cosmography with the Einstein Telescope
}

\author{
B S Sathyaprakash ${ }^{1}$, B F Schutz ${ }^{2}$ and C Van Den Broeck ${ }^{3}$ \\ ${ }^{1}$ School of Physics and Astronomy, Cardiff University, Cardiff, CF24 3AA, UK \\ ${ }^{2}$ Max Planck Institute for Gravitational Physics, The Albert Einstein Institute, \\ Am Mühlenberg 1, Golm, D-14476, Germany \\ ${ }^{3}$ Nikhef - National Institute for Subatomic Physics, Science Park 105, 1098 XG Amsterdam, \\ The Netherlands \\ E-mail: B.Sathyaprakash@astro.cf.ac.uk,B.F.Schutz@aei.mpg.de and vdbroeck@nikhef.nl
}

Received 13 May 2010, in final form 3 August 2010

Published 27 September 2010

Online at stacks.iop.org/CQG/27/215006

\begin{abstract}
The Einstein Telescope, a third-generation gravitational-wave detector under a design study, could detect millions of binary neutron star inspirals each year. A small fraction of these events might be observed as gamma-ray bursts, helping to measure both the luminosity distance $D_{\mathrm{L}}$ to and redshift $z$ of the source. By fitting these measured values of $D_{\mathrm{L}}$ and $z$ to a cosmological model, it would be possible to infer the dark energy equation of state to within $1.5 \%$ without the need to correct for errors in $D_{\mathrm{L}}$ caused by weak lensing. This compares favourably with $0.3-10 \%$ accuracy that can be achieved with the Laser Interferometer Space Antenna (where weak lensing will need to be dealt with) as well as with dedicated dark energy missions that have been proposed, where $3.5-11 \%$ uncertainty is expected.
\end{abstract}

PACS numbers: $04.30 . \mathrm{Db}, 04.25 . \mathrm{Nx}, 04.80 . \mathrm{Nn}$, 95.55.Ym

(Some figures in this article are in colour only in the electronic version)

\section{Introduction}

Standard candles are used to measure the geometry and dynamics of the Universe. A standard candle is a source whose intrinsic luminosity $L$ can be inferred from the observed properties such as its spectral content, time variability of the flux of the radiation it emits, etc. Since the observations also measure the apparent luminosity $F$, one can deduce the luminosity distance $D_{\mathrm{L}}$ to a standard candle from $4 \pi D_{\mathrm{L}}^{2}=L / F$. If the redshift $z$ to the source is known, then from a population of such sources it will be possible to measure the cosmological parameters since the luminosity distance is related to the redshift, in a flat Universe, via

$$
D_{\mathrm{L}}(z)=\frac{c(1+z)}{H_{0}} \int_{0}^{z} \frac{\left(1+z^{\prime}\right)^{-3 / 2} \mathrm{~d} z^{\prime}}{\left[\Omega_{\mathrm{M}}+\Omega_{\Lambda}\left(1+z^{\prime}\right)^{3 w}\right]^{1 / 2}},
$$


where $H_{0}$ is the Hubble constant, $\Omega_{\mathrm{M}}$ and $\Omega_{\Lambda}$ are the dark matter and dark energy densities, respectively, at the present time and $w$ is the dark energy equation-of-state parameter.

There is no unique standard candle in astronomy that works for all distances. A distance scale is built by using several candles, each of which works over a limited range of distance. For instance, the method of parallax can determine distances of a few kpc, Cepheid variables up to $10 \mathrm{Mpc}$, the Tully-Fisher relation up to tens of $\mathrm{Mpc}$, the $D_{n}-\sigma$ relation up to hundreds of Mpc and type Ia supernovae up to only a few redshifts [1]. This way of building a distance scale has been referred to as the cosmic distance ladder. For cosmography, a proper calibration of distances to high redshift galaxies is based on the mutual agreement between the different rungs of this ladder. It is critical that each of the rungs is calibrated with an error as small as possible.

\section{Self-calibrating standard sirens of gravity}

Cosmologists have long sought standard candles that can work over large distance scales without being dependent on the lower rungs of the cosmic distance ladder. In 1986, it was discovered [2] that gravitational astronomy can provide such a candle, or, more appropriately, a standard siren, in the form of an inspiraling compact binary consisting of neutron stars (NSs) and black holes (BHs). This method needs no calibration at all, relying purely on the modelling of the two-body problem in general relativity. Gravitational-wave observations, therefore, provide not only a powerful distance measuring tool but also a useful check on other distance ladders.

In simple terms, in the inspiral phase of a compact binary, the amplitude of gravitational waves depends on the ratio of a certain combination of the component masses called the chirp mass and the luminosity distance. Both of these can be measured for a chirping signal—a signal whose frequency increases by a measurable amount during its observation. For such sources one can directly infer the luminosity distance. In reality, the response of an interferometer to such a signal, in an approximation that keeps only the dominant signal harmonic at twice the orbital frequency, is given by

$$
h(t)=\frac{4 A(\theta, \varphi, \psi, \iota) \nu M[\pi M F(t)]^{\frac{2}{3}} \cos \Phi(t)}{D_{\mathrm{L}}} .
$$

Here, $0 \leqslant A \leqslant 1$ is a numerical factor that depends on the location of the binary on the sky and its orientation relative to the detector; $M$ and $v$ are, respectively, the binary's total mass and symmetric mass ratio; $\Phi(t)$ and $F(t)$ are the signal's phase and frequency; $D_{\mathrm{L}}$ is the luminosity distance; $(\theta, \varphi)$ gives the source's location on the sky; $\iota$ is the orientation of the system relative to the line of sight; and $\psi$ is the wave's polarization angle. In the case of non-spinning binaries in quasi-circular orbits, an inspiral signal is characterized by nine parameters in all $\left(M, v, t_{0}, \Phi_{0}, \theta, \varphi, \psi, \iota, D_{\mathrm{L}}\right)$. Here, $t_{0}$ and $\Phi_{0}$ are the fiducial parameters defining the epoch when the signal's frequency reaches a certain value and its phase at that epoch. In this approximation, the signal amplitude depends only on the chirp mass $\mathcal{M}$ defined by $\mathcal{M}=v^{3 / 5} M$ and not separately on the two mass parameters.

The signal's phase has been computed to high order in post-Newtonian theory [3] and depends only on the two mass parameters; here we go to $3.5 \mathrm{PN}$. One can therefore employ matched filtering to extract the signal and to measure the two mass parameters $(M, v)$ as well as the two fiducial parameters $\left(t_{0}, \Phi_{0}\right)$. Note that for a source at a redshift $z$ the signal's frequency will be redshifted to $F \rightarrow F /(1+z)$ but the mappings $M \rightarrow(1+z) M$ and $D_{\mathrm{L}} \rightarrow(1+z) D_{\mathrm{L}}$ leave the signal invariant. Thus, a source of intrinsic total mass $M_{i}$ will appear to be a binary 
of total mass $M=(1+z) M_{i}$. One must optically identify the host galaxy and measure its redshift to deduce its intrinsic mass $M_{i}$.

\section{Multi-messenger cosmology with binary neutron stars and GRBs}

In general, the response of a single interferometer will not be sufficient to disentangle the luminosity distance from the angular parameters. An optical identification of the source can determine the direction to the source (and its redshift), leaving three unknown parameters $\left(\psi, \iota, D_{\mathrm{L}}\right)$. Additionally, if the signal is associated with a gamma-ray burst (GRB), then the source's orbital plane will be perpendicular to the line of sight, implying that $\iota \simeq 0$ and $\psi \simeq 0$. Thus, for inspirals detected in coincidence with GRBs, a single detector is good enough to measure the luminosity distance. Multi-messengers like GRBs can, therefore, make precision cosmography possible, without the need to build a cosmic distance ladder.

For signals not associated with a GRB, $D_{\mathrm{L}}$ can be measured if the source lasts long enough to cause a modulation in the signal's frequency due to the motion of the detector relative to the source, as would be the case for the Laser Interferometer Space Antenna (LISA). If the signal lasts for only a short time, as would be the case for ground-based detectors, one would need a network of three detectors to measure $D_{\mathrm{L}}$.

Over the next two decades GW detectors could provide a new tool for cosmology. The Laser Inteferometer Gravitational-Wave Observatory (LIGO) in the USA and Virgo in Europe have reached design goals for their initial operation. Recent science runs of LIGO and Virgo have begun to impact our understanding of astrophysical sources and phenomena. Both are now getting ready to upgrade to advanced sensitivities by 2014 and are expected to detect $\sim 40$ binary neutron star (BNS) mergers each year [4, 5]. Redshift could be measured to a (small) number of events associated with GRBs and might allow the measurement of the expansion rate of the Universe in the $\sim 500 \mathrm{Mpc}$ range, where optical data is scarce [6, 7]. Observation by the LISA of extreme mass ratio inspirals could measure the Hubble constant pretty accurately [8]. The LISA will also observe binary super-massive BH mergers with signal-to-noise ratios (SNRs) of several thousands at $z \sim 1$, enabling the measurement of the dark energy equation of state to within several percent $[9,10]$.

In the rest of this paper we will discuss how well it might be possible to constrain cosmological parameters with the Einstein Telescope (ET) - a third-generation ground-based interferometer that is currently under a design study $[11,12]$. The ET is envisaged to be ten times more sensitive in amplitude than the advanced ground-based detectors, covering a frequency range of $1-10^{4} \mathrm{~Hz}$. Achieving the sensitivity of the ET will pose challenges in mitigating gravity gradient, thermal and quantum noises but careful analysis shows that the technology might be within reach in the next decade [13]. One possible topology for the ET could be an equilateral triangle, $10 \mathrm{~km}$ on a side, allowing the operation of three V-shaped interferometers at a single site $[14,15]$. A network of such detectors might be available over the next 15-20 years, but we will explore how accurately it might be possible to measure the cosmological parameters with a single such ET.

\subsection{Distance reach of the ET for neutron star binaries and event rates}

For a fixed SNR $\rho_{0}$, the distance up to which an inspiral signal could be detected in the ET is given by

$$
D_{\mathrm{L}}\left(\rho_{0}\right)=\frac{A^{\prime}(\theta, \varphi, \iota, \psi) \mathcal{M}^{5 / 6}}{\rho_{0}}\left[\int_{F_{\mathrm{s}}}^{F_{\mathrm{lso}}} \frac{f^{-7 / 3}}{S_{h}(f)} \mathrm{d} f\right]^{1 / 2},
$$




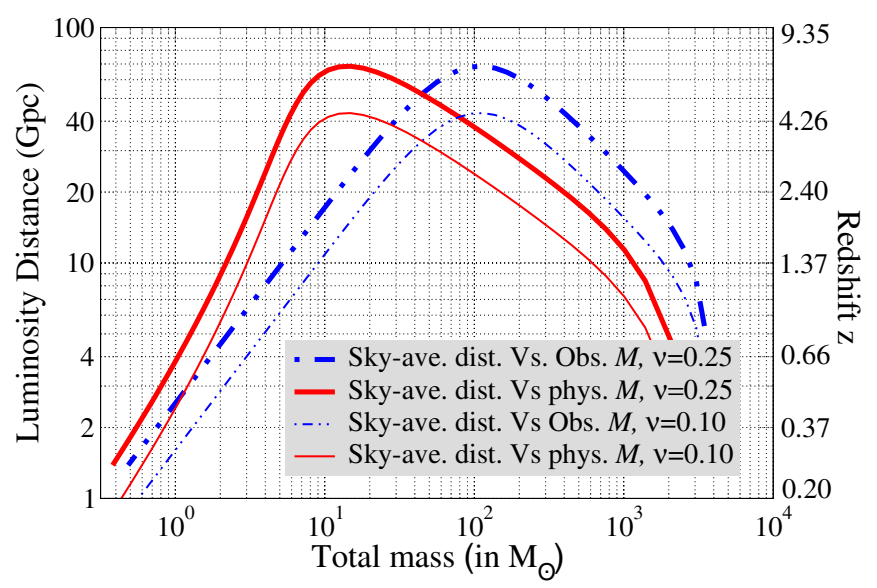

Figure 1. Range of the ET for inspiral signals from binaries as a function of the intrinsic (red solid line) and the observed (blue dashed line) total mass. We assume that a source is visible if it produces an SNR of at least 8 in the ET.

where $S_{h}(f)$ is the one-sided noise power spectral density (PSD), which we take to be the 'ET-B' PSD as in figure 1 of [15]; we also assume the $10 \mathrm{~km}$ triple Michelson setup as explained in that reference. $F_{\mathrm{S}}$ is a frequency below which the accumulated SNR is negligible because the PSD rises far faster than the signal spectrum and $F_{\text {lso }}$ is the signal frequency corresponding to the last stable orbit of the binary, taken to be $F_{\text {lso }}=1 /\left(6^{3 / 2} \pi M\right)$. The quantity $A^{\prime}$ is an appropriate combination of the function $A$ in equation (2) and the antenna pattern functions of the three independent Michelson interferometers. In computing the distance reach of the ET one can take an average of $A^{\prime}$ over sky position and set $\iota=\psi=0$, again assuming relatively strong beaming of GRBs; the corresponding root-mean-square (rms) value is $A_{\mathrm{rms}}^{\prime}=\sqrt{2 / 5} A_{\mathrm{opt}}^{\prime}$, where $A_{\mathrm{opt}}^{\prime}$ is the value for an optimally oriented and positioned system [16]. However, we note that GRBs may have beaming angles up to $\sim 40^{\circ}$, i.e. $\iota \sim 20^{\circ}$ [17]. If one averages over all angles $(\theta, \phi, \psi, \iota)$ but with the constraint $\iota<20^{\circ}$, then the rms value becomes $A_{\text {rms }}^{\prime}=\alpha A_{\text {opt }}^{\prime}$ with $\alpha \simeq 0.614$, which is barely different from the case $\iota=\psi=0$. The distance up to which the ET might detect signals from an inspiraling BNS with an SNR of 8 is shown in figure 1 as a function of the observed total mass (blue dashed lines). We assume a cosmological model in which $H_{0}=70 \mathrm{~km} \mathrm{~s}^{-1} \mathrm{Mpc}^{-1}, \Omega_{\mathrm{M}}=0.27$, $\Omega_{\Lambda}=0.73$ and $w=-1$, which allows us to convert distances to redshifts by inverting equation (1), and which we have used to convert from the observed masses in figure 1 to the intrinsic masses (red solid lines).

By extrapolating the rate of BNS inspirals expected in advanced detectors to the ET, whose distance reach for a BNS is $z \simeq 2$ or $D_{\mathrm{L}} \simeq 16 \mathrm{Gpc}$, one might expect $4 \times 10^{7}$ events per year. Of course, this naive extrapolation does not give the correct rate as it does not include the cosmological evolution of compact binaries. For our purposes, however, even if the rate is an order of magnitude lower it does not matter. As an aside, legitimate concern has been raised that this high event rate may lead to a confusion background [18]. As it turns out, the $P S D$-weighted signals are actually quite short and will not tend to have significant overlap with each other [19].

As noted earlier, in order for BNS inspirals to be useful for cosmography, it is essential that their location on the sky and redshift are determined separately. If, as suspected, BNSs are 
progenitors of short-hard GRBs [17], then it might be possible to make a coincident detection of a significant subset of the events in GW and electromagnetic (EM) windows.

Since GRBs are believed to be beamed with beaming angles of order $40^{\circ}$, we assume that only a small fraction $\left(\sim 10^{-3}\right)$ of binary coalescences will have a GRB or other EM afterglows that will help us to locate the source on the sky and measure its redshift. Eventually, we will be limited by the number of short-hard GRBs observed by detectors that might be operating at the time. As a conservative estimate, we assume that about 1000 BNS mergers will have EM counterparts over a 5 year period. For definiteness, we consider only BNS mergers and take these to have component masses $(1.4,1.4) M_{\odot}$.

\subsection{Measurement accuracy of cosmological parameters}

How well would we measure cosmological parameters with a catalogue of such sources? To answer this question we simulated 5190 realizations of the catalogue containing 1000 BNS coalescences with known redshift and sky location, but with the luminosity distance subject to statistical errors from $\mathrm{GW}$ observation and weak lensing. We assumed that all sources were in the redshift range $0 \leqslant z \leqslant 2$, distributed uniformly (i.e. with constant comoving number density) throughout this redshift range. The luminosity distance to the source was computed by assuming an FRW cosmological model with $H_{0}=70 \mathrm{~km} \mathrm{~s}^{-1} \mathrm{Mpc}^{-1}, \Omega_{\mathrm{M}}=0.27, \Omega_{\Lambda}=0.73$ and $w=-1$, but the measured distance was drawn from a Gaussian distribution whose width $\sigma_{D_{\mathrm{L}}}$ was determined by the quadrature sum of the errors due to weak lensing and GW observation. Weak lensing error in $D_{\mathrm{L}}$ was assumed to be $5 \%$ at $z=1$ [20] and linearly extrapolated to other redshifts. The GW observational error was estimated from the covariance matrix $C_{k m}$ of the parameter space of the unknown signal parameters $p_{k}$ :

$$
C_{k m}=\Lambda_{k m}^{-1}, \quad \Lambda_{k m}=\left\langle h_{k}, h_{m}\right\rangle, \quad h_{k}=\frac{\partial h}{\partial p_{k}} .
$$

Here, the angular brackets denote the scalar product, which, for any two functions $a(t)$ and $b(t)$, is defined as

$$
\langle a, b\rangle=4 \Re \int_{0}^{\infty} \frac{\mathrm{d} f}{S_{h}(f)} A(f) B^{*}(f),
$$

where $A$ and $B$ are the Fourier transforms of the functions $a(t)$ and $b(t)$, respectively, and $S_{h}(f)$ is the ET noise PSD. In computing the covariance matrix, we made a distinction between the following two cases.

- If the beaming is so strong that one can assume $\iota=\psi=0$ for all practical purposes, then the parameters to be estimated are

$$
p_{k}=\left(M, v, t_{0}, \Phi_{0}, D_{\mathrm{L}}\right) \text {. }
$$

At a given redshift, the resulting rms uncertainty on $D_{\mathrm{L}}$ was then averaged over sky position.

- Allowing for beaming angles as wide as $\iota \sim 20^{\circ}$, the binary's orientation $(\psi, \iota)$ also needs to be taken into account:

$$
p_{k}=\left(M, v, t_{0}, \Phi_{0}, D_{\mathrm{L}}, \psi, \iota\right)
$$

In this case the rms errors were averaged over all angles $(\theta, \phi, \psi, \iota)$ but with the constraint $\iota<20^{\circ}$.

We then fitted each realization of the source catalogue to the cosmological model given in equation (1), using the Levenberg-Marquardt algorithm [21, 22], in order to find a set of best fit parameters. It turns out that a catalogue of 1000 sources is not quite enough for an 


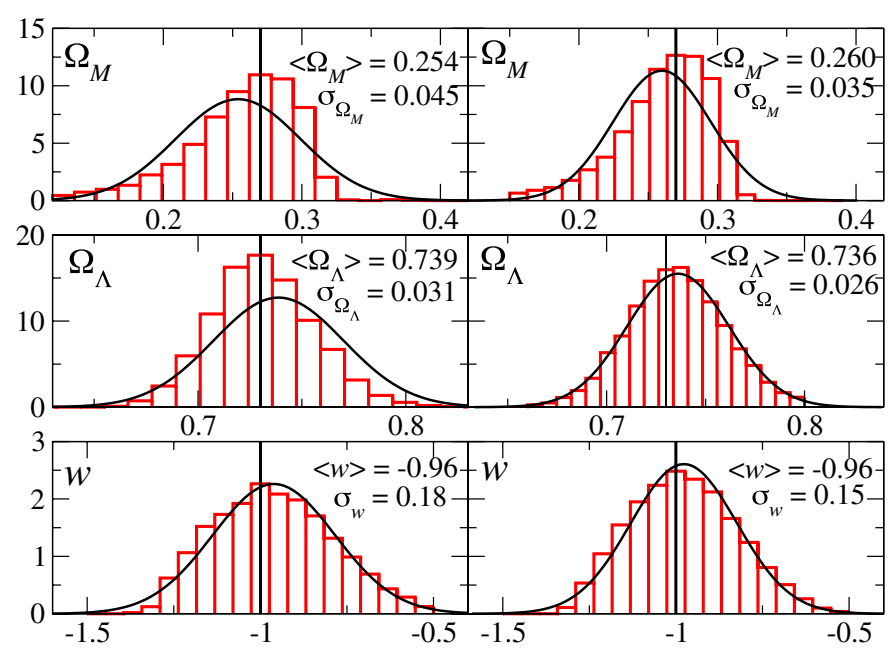

Figure 2. The plot shows the distribution of errors in $\Omega_{M}, \Omega_{\Lambda}$ and $w$, obtained by fitting 5190 realizations of a catalogue of BNS merger events to a cosmological model of the type given in equation (1), with three free parameters. The fractional $1-\sigma$ widths of the distributions $\sigma_{\Omega_{M}} / \Omega_{\mathrm{M}}$, $\sigma_{\Omega_{\Lambda}} / \Omega_{\Lambda}$ and $\sigma_{w} /|w|$, respectively, are $18 \%, 4.2 \%$ and $18 \%$ (with weak lensing errors in $D_{\mathrm{L}}$, left panels) and $14 \%, 3.5 \%$ and $15 \%$ (if weak lensing errors can be corrected, right panels).

accurate determination of all the parameters. However, assuming that $H_{0}$ is known accurately, the algorithm gave the best fit parameters in $\left(\Omega_{\mathrm{M}}, \Omega_{\Lambda}, w\right)$ for each of the 5190 realizations. By the time ET is operational, a network of advanced detectors will have been active over a number of years, possibly measuring $H_{0}$ down to a percent or better over such a time span [7]. Using extreme mass ratio inspirals seen in the LISA, one can also measure $H_{0}$ to better than a percent [8], or down to a fraction of a percent judging from relative distance errors for supermassive binary $\mathrm{BH}$ coalescences [10]. Taking $H_{0}$ to be the only free parameter and with only 50 sources up to a redshift of $z=0.5$, the ET itself would achieve an accuracy of $0.55 \%$ (using the kind of estimation explained here). For sufficiently low-redshift sources, the rest of cosmology is not very important. Having determined $H_{0}$ in this way, the higher redshift sources can then be used to explore the cosmological parameter space more fully. In this way, GW astronomy will provide an independent measure of cosmography.

Thus, the ET itself can use sources in the low-redshift Universe to measure $H_{0}$ with an error that is negligible compared to the uncertainties on the other parameters obtained from all sources combined, as we will see below. For the purposes of this paper we will consider $H_{0}$ essentially known.

Let us first discuss some results in the case of strong beaming $(\iota=\psi=0)$; in the next section we will relax this assumption. The distributions $\mathcal{P}$ of the parameters obtained in the way we described above are shown in figure 2 , where the vertical line is at the true value of the relevant parameter. Figure 3 shows a scatter plot of the recovered values of the dark energy density and dark energy equation of state when not correcting for weak lensing errors.

The relative $1-\sigma$ errors in $\Omega_{\Lambda}, \Omega_{\mathrm{M}}$ and $w$ are $4.2 \%, 18 \%$ and $18 \%$ (with weak lensing) and $3.5 \%, 14 \%$ and $15 \%$ (with weak lensing errors corrected). Although $\mathcal{P}(w)$ is quite symmetric, $\mathcal{P}\left(\Omega_{\mathrm{M}}\right)$ and $\mathcal{P}\left(\Omega_{\Lambda}\right)$ are both skewed and their mean values are slightly off the true values. The medians, however, are coincident with the true values. 


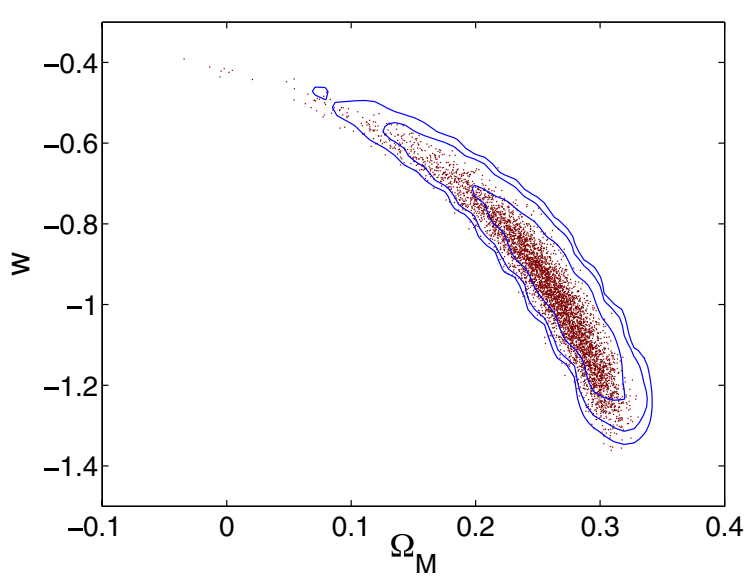

Figure 3. Scatter plot of the retrieved values for $\left(\Omega_{\Lambda}, w\right)$, with 1- $\sigma, 2-\sigma$ and 3- $\sigma$ contours, in the case where weak lensing is not corrected.

In addition to $H_{0}$ if $\Omega_{\Lambda}$ is also known (or, equivalently, if $\Omega_{M}+\Omega_{\Lambda}=1$ ), then one can estimate the pair $\left(\Omega_{\mathrm{M}}, w\right)$ more accurately, with $1-\sigma$ errors in $\Omega_{\mathrm{M}}$ and $w$ of $9.4 \%$ and $7.6 \%$ (with weak lensing) and $8.1 \%$ and $6.6 \%$ (with lensing errors corrected). Finally, if $w$ is the only parameter unknown, it can be measured to an even greater accuracy with 1- $\sigma$ errors of $1.4 \%$ (with weak lensing) and $1.1 \%$ (with lensing errors corrected) ${ }^{4}$.

\subsection{Effect of unknown orientation and polarization}

In the previous section our study neglected the effect of different inclinations of the orbit to the line of sight. Varying the inclination has two distinct effects. On the one hand, as noted in [7], due to the strong correlation between the luminosity distance and the inclination, the estimation of the luminosity distance could get corrupted. On the other hand, binaries that are not face-on are, in general, elliptically polarized and have a non-zero polarization angle. Since the polarization angle is correlated with the luminosity distance, there could be further degradation in the estimation of the luminosity distance.

In this section we relax the condition that the inclination of the orbit is precisely known. However, we will restrict the inclination of the binary's angular momentum with the line of sight to be within $20^{\circ}$. We will also assume that the radiation is described by an arbitrary polarization angle. Since the sky position is still assumed to be known, this gives us a $7 \times 7$ covariance matrix with a revised estimate for the error in the luminosity distance. As before, we construct catalogues of binary coalescence events but with the luminosity distance now drawn from a Gaussian distribution with revised widths. We fit each catalogue to a cosmological model and then repeat the exercise 5190 times to estimate the accuracy with which the various cosmological parameters can be measured.

As expected, the parameter measurements get worse if we assume two or more parameters to be unknown. For instance, errors in the estimation of $\Omega_{M}, \Omega_{\Lambda}$ and $w$ are, respectively,

4 At this point we note that in contemporary cosmology, $w$ is determined mainly through SNIa observations using $\mathrm{CMB}$ data as prior to 'fix' the other parameters. The CMB constraint on $w$ is extremely weak. If one were to use $\mathrm{CMB}$ results as a prior for GW measurements, one would obtain an independent measurement of $w$. We stress once again that, unlike supernovae, GW standard sirens do not need any external calibration. A detailed discussion will be presented in forthcoming work [23]. 
more than $100 \%, 24 \%$ and $47 \%$ with weak lensing and more than $100 \%, 21 \%$ and $43 \%$, if weak lensing can be corrected. Similarly, if $\Omega_{\Lambda}$ is assumed to be known, then the errors in the estimation of $\Omega_{M}$ and $w$ are, respectively, $12 \%$ and $9.5 \%$ if weak lensing is uncorrected for and $11 \%$ and $9.2 \%$ if weak lensing can be corrected. However, the results are more or less the same if dark energy parameter $w$ is the only unknown quantity. Even when the inclination and polarization angles are taken as free parameters, but the inclination angle is restricted to within $20^{\circ}$, the error in the estimation of $w$ is $1.4 \%$ with weak lensing and $1.3 \%$ if weak lensing can be corrected.

\subsection{Comparison with other experiments and space missions}

These results are better than what could be achieved with the LISA, which in estimating $w$ as a single free parameter may reach an accuracy of $0.3-10 \%$ depending on the systems available, but under the condition that weak lensing effects can at least partially be subtracted [10], otherwise LISA's error will be much larger (in the order of 20\%). Our results where multiple parameters are estimated at once are also competitive with what is deemed possible with proposed dedicated dark energy missions; see, e.g., the report of the Dark Energy Task Force [24], who expect to see $w$ errors between $3.5 \%$ and $11 \%$.

In the above, we computed uncertainties for at most three free parameters. When $\left(H_{0}, \Omega_{\mathrm{M}}, \Omega_{\Lambda}, w\right)$ are all estimated at the same time, the relative $1-\sigma$ errors come out to be $13 \%, 37 \%, 36 \%$ and $23 \%$, respectively. We stress once again that the ET can use a relatively small number of sources at low redshifts to obtain $H_{0}$ with almost negligible uncertainty; this parameter can then be considered fixed to study $\left(\Omega_{\mathrm{M}}, \Omega_{\Lambda}, w\right)$ over a much larger redshift range where the dynamical history of the Universe truly comes to bear.

\section{Conclusions}

In this study, we have shown that using a population of BNSs, the ET can measure the dark energy equation-of-state parameter $w$ (assuming this is the only unknown parameter) to within about $1.5 \%$. This precision is achievable even if it is not possible to correct for the errors in the luminosity distance caused by weak lensing and the unknown inclination and polarization angles of the binary.

The results of our simulation are quite encouraging but further work is needed to confirm the usefulness of GW standard sirens in precision cosmology. Let us mention some that are currently being pursued. Spins of component stars can be legitimately neglected in the case of NSs (and hence in BNSs) but not for BHs. The modulation in the signal caused by the spin of the black hole can improve parameter estimation accuracies [25]. We assumed, for simplicity, that all our sources are BNS systems with masses $(1.4,1.4) M_{\odot}$. In reality, the catalogue will consist of a range of NS and $\mathrm{BH}$ masses. A more realistic Monte Carlo simulation would draw binaries from the expected population rather than the same system, some of which (e.g. more massive systems) would lead to better, but others to worsened, parameter accuracies. The signal contains additional features, such as other harmonics of the orbital frequency than the second harmonic considered in this work, and the merger and ringdown signals. These are important for heavier systems and could potentially reduce the errors. Also, a recent study [26] has shown that weak lensing effects on the measurement of the luminosity distance could be reduced by $50 \%$, which should make for improved parameter accuracies.

These factors are currently being taken into account to get a more reliable estimation of the usefulness of the ET in precision cosmography. 


\section{References}

[1] Freedman W L et al 2001 Astrophys. J. 553 47-72 (arXiv:astro-ph/0012376)

[2] Schutz B 1986 Nature 323 310-1

[3] Blanchet L, Faye G, Iyer B R and Joguet B 2002 Phys. Rev. D 65061501 (arXiv:gr-qc/0105099)

[4] Kopparapu R et al 2008 Astrophys. J 675 1459-67

[5] Abadie J et al (LIGO Scientific) 2010 Class. Quantum Grav. 27173001 (arXiv:1003.2480)

[6] Dalal N, Holz D E, Hughes S A and Jain B 2006 Phys. Rev. D 74063006 (arXiv:astro-ph/0601275)

[7] Nissanke S, Hughes S A, Holz D E, Dalal N and Sievers J L 2009 arXiv:0904.1017

[8] MacLeod C L and Hogan C J 2008 Phys. Rev. D 77043512 (arXiv:0712.0618)

[9] Holz D and Hughes S 2005 Astrophys. J 629 15-22 (arXiv:astro-ph/0504616)

[10] Arun K, Iyer B, Sathyaprakash B, Sinha S and Van Den Broeck C 2007 Phys. Rev. D 76 (arXiv:0707.3920)

[11] Einstein Telescope project https://www.et-gw.eu/et/

[12] Punturo M et al 2010 Class. Quantum Grav. 27084007

[13] Hild S, Chelkowski S and Freise A 2008 arXiv:0810.0604

[14] Freise A, Chelkowski S, Hild S, Del Pozzo W and Perreca A 2009 Class. Quantum Grav. 26085012

[15] Freise A, Hild S, Komiya K, Strain K, Viceré A, Barsuglia M and Chelkowksi S 2009 arXiv:0908.0353

[16] Damour T, Iyer B R and Sathyaprakash B S 2000 Phys. Rev. D 62084036 (arXiv:gr-qc/0001023)

[17] Nakar E 2007 Phys. Rep. 442 166-236 (arXiv:astro-ph/0701748)

[18] Regimbau T and Hughes S 2009 Phys. Rev. D 79062002

[19] Regimbau T 2010 private communication

[20] Kocsis B, Frei Z, Haiman Z and Menou K 2006 Astrophys. J. 637 27-37

[21] Levenberg K 1944 Q. Appl. Math. 2 164-8

[22] Marquardt D 1963 SIAM J. Appl. Math. 11 431-41

[23] Zhao W, Van Den Broeck C, Baskaran D and Li T G F 2010 Determination of dark energy by the Einstein Telescope: comparing with CMB, BAO and SNIa observations arXiv:1009.0206

[24] Albrecht A et al 2006 arXiv:astro-ph/0609591

[25] Vecchio A 2004 Phys. Rev. D 70042001 (arXiv:astro-ph/0304051)

[26] Shapiro C, Bacon D, Hendry M and Hoyle B 2009 arXiv:0907.3635 